\title{
Clinical features of women's depending of paroxetine (paxil) and their treatment
}

\author{
Nadir A. Aliyev ${ }^{*}$ and Zafar N. Aliyev ${ }^{2}$ \\ ${ }^{1}$ Department of Psychiatry and Addiction Institute of Postgraduate Medical named A. Aliyev of Azerbaijan Republic of the Ministry of the Health, Baku City, \\ Azerbaijan Republic \\ ${ }^{2}$ Department of Psychiatry in Azerbaijan Medical State University, Baku City, Azerbaijan Republic
}

\begin{abstract}
Objective: The aim of this work was study of clinical features of women's depending of Paxil.

Materials and methods: A structured clinical interview, according to the checklist of diagnostic criteria of ICD-10 (Psychoactive Substance Use Syndromes Module). Ten women patients depending of paxil were studied. Results: It was found that the main clinical features depending on Paxil in women are: the prevalence of the clinical picture of mental disorders such as psychosis (atypical form of delirium) and non-psychotic, excessive agitation, suicidal attempts, prone to aggressive actions to themselves or others, autonomic, somatic, and neurological and other disorders.
\end{abstract}

Conclusion: Our data suggest that treatment depending of paxil must be direction to psychopathological sings, which appeared clinical picture of the illness.

\section{Introduction}

The World Health Organization [1] compares the depression with the epidemic that has engulfed the whole humanity, depression has moved into first place in the world among the causes of absenteeism, the second - of disease, leading to disability. If no action is taken, by 2020 depression will paralyze the economic life in both developed and developing countries. WHO predicts that by 2020, depression will rank first in the world among all diseases, ahead of today's leaders, infectious and cardiovascular diseases. Today it is the most common disease that affects women.

Every year about 150 million people in the world are denied disability because of depression. Only the U.S. economy, it causes annual cost of more than 50 billion dollars in the United States, according to the American Psychiatric Association, depression affects 15 million people. Other studies estimate the prevalence of depressive disorders is much higher - more than $26 \%$ of women and $12 \%$ among men. To date, the U.S. depression - the second "by popularity» reason that hospitals give sick-leave certificate, Sweden - first.

About $80 \%$ of antidepressants in most Western European countries, USA, Canada prescribed by general practitioners, not psychiatrists. In Russia and other former Soviet republics formally primary care physicians can prescribe such drugs (except "heavy", causing side effects), but more often they do not have the necessary experience or qualifications.

Women have depression 2 times more likely than men (20-26\% vs. $8-12 \%$, respectively). From 45 to $60 \%$ of all suicides in the world make depressed patients. According to forecasts, by 2020 depression will be the killer number 1 .

When a single episode of depression likelihood of recurrence is $50 \%-70 \%$ in the second, third is already $90 \%$.
Today in the world used about 100 drugs with thymoleptic activity, most of them are also registered in our country. Because of the transition to a market economy in pharmacies increasingly appear unfamiliar or not previously known means, handouts which report very high or almost universal effectiveness for depression. Understanding of this is not easy. Comparative lack of objective information, frequent errors in the readings and how to apply, and as a consequence, lack of proper result cause a certain negativity to new drugs among practitioners. Despite significant progress, the problem of pharmacotherapy of depression is still far from being resolved. Clinical practice and numerous comparative studies show that the newly synthesized agents generally do not appear to be more efficient than the classic antidepressants [2].

Antidepressants (thymoleptics) are a group of remedies and psychoanaleptic have predominant influence on pathologically depressed mood or depressive affect. In healthy individuals they do not cause any improvement in mood.

Selective serotonin reuptake inhibitors (SSRIs) and tricyclic antidepressants have comparable efficacy, although in some cases antidepressant effect develops gradually after 6-8 weeks of therapy. Issues related to the mechanism of action, the main indications for use, general guidelines for the management of patients, comparing SSRIs

Correspondence to: Nadir A. Aliyev, Department of Psychiatry and Addiction Institute of Postgraduate Medical named A. Aliyev of Azerbaijan Republic of the Ministry of the Health, Baku City, U. Chagibekov Street, 46/50, F. 1. Baku P.O. AZ0010, Azerbaijan Republic, Tel: +86 25663 7551; E-mail: aliyevnadir@yahoo.com

Key words: depending, paxil, clinic, women

Received: May 13, 2016; Accepted: June 14, 2016; Published: June 17, 2016 
with heterocyclic and other antidepressants are widely reflected in the literature. Therefore, we will pass this.

One of the representatives of the group SSRI antidepressants is paroxetine (synonyms: Paxil, Reksetin, Seroxat). Paxil is manufactured by GlaxoSmithKline - GSK. This drug is widely used both in Russia and in other countries for the treatment of depression, panic, obsessivecompulsive, generalized anxiety and post-traumatic stress disorder.

The fact that Paxil is addictive, is discussed in the largest Russianspeaking forums (http://neuroleptic.ru/, http://nevrozu.net/, http:// www.pan-at.com/). In the United States there were repeatedly given claims to ban sales of the drug Paxil GSK due to strong withdrawal. GSK Company pleaded guilty and paid compensation to the victims.

Thus, analysis of world literature data on clinical trials of Paxil, depending on general and in particular for women, and methods of treating it, is missing, and that was the basis for this work.

Objective- to study the clinical features depending on Paxil in women and development of methods of treatment.

\section{Patients and methods}

Twenty women were under the supervision of the Centre of Mental Health of the Ministry of the Health of the Azerbaijan Republic in a private manner. Work was carried out from January to December 2012.

Diagnosis of dependence of Paxil has been set, according to the checklist of diagnostic criteria of ICD-10 (Psychoactive Substance Use Syndromes Module) [3]. Diagnostic criteria checklist consists of nine questions relating to the time period immediately preceding the beginning of the current period of treatment. About syndrome depending indicating the presence of three or more symptoms of 1 , 2, 3, 5, 7 and 9 (The ICD-10 Symptom Checklist for Mental Disorders World Health Organization 2004) [3]. From all patients were obtained 5 or more positive responses on the symptoms. This study is not commercially sponsored. For the patients of the present study was prepared Paxil (40-60 mg/day), they worked, had secondary or university education, were married. Age of patients ranged from 30 to 55 years (standart deviation $37,5 \pm 5,6$ ). Duration of taking the Paxil was $4-5$ years. All examined patients suffered from major depressive disorder, current episode - severe without psychotic symptoms (F33.2). Examined patients prescribed Paxil in foreign countries. In anamnesis data, elation, hyperactivity, which could meet the criteria for mania (F30.1 and F30.2x), was not observed. F33 Recurrent depressive disorder: A disorder characterized by repeated episodes of depression as described for depressive episode (F32.-), without any history of independent episodes of mood elevation and increased energy (mania). There may, however, be brief episodes of mild mood elevation and overactivity (hypomania) immediately after a depressive episode, sometimes precipitated by antidepressant treatment. The more severe forms of recurrent depressive disorder (F33.2 and F33.3) have much in common with earlier concepts such as manic-depressive depression, melancholia, vital depression and endogenous depression. The first episode may occur at any age from childhood to old age, the onset may be either acute or insidious, and the duration varies from a few weeks to many months. The risk that a patient with recurrent depressive disorder will have an episode of mania never disappears completely, however many depressive episodes have been experienced. If such an episode does occur, the diagnosis should be changed to bipolar affective disorder (F31.-).

Includes: recurrent episodes of: depressive reaction; psychogenic depression; reactive depression; seasonal depressive disorder.

F33.2 Recurrent depressive disorder, current episode severe without psychotic symptoms. A disorder characterized by repeated episodes of depression, the current episode being severe without psychotic symptoms, as in F32.2, and without any history of mania. Endogenous depression without psychotic symptoms; Major depression, recurrent without psychotic symptoms; Manic-depressive psychosis, depressed type without psychotic symptoms; Vital depression, recurrent without psychotic symptoms [4].

All patients provided written consent information after a full explanation of the research protocol. Our study has been conducted in accordance with the Declaration of Helsinki and according to requirements of Azerbaijan Low of Healthy and Azerbaijan Low about Psychiatric service. We attest that all application subject protection guidelines and regulation were followed in the conduct of the research.

\section{Results and discussion}

Examined patients treated in mental health center about the fact that they cannot stop taking Paxil. We tried to stop them to taking the medicine, according to the manufacturer's instructions. Company recommended the following scheme of cancellation: reducing the daily dose by $10 \mathrm{mg}$ at weekly intervals; after the dose of $20 \mathrm{mg} /$ day (or $10 \mathrm{mg} /$ day. in children and adolescents ) patients continue to take this dose for 1 week. And only after this drug overturned completely. If withdrawal symptoms occur during dose reduction or drug discontinuation, it is advisable to renew reception previously prescribed dose. Subsequently, the physician may continue decreasing the dose, but more slowly. However, this scheme discontinuation of Paxil caused severe withdrawal, accompanied by psychotic (visual and auditory hallucinations), affective phenomena (depressed mood tinged with gloom, melancholy, gloomy discontent with spiteful irritability); manifested a combination of melancholy or sad mood, anxiety irritability, anger turning into aggressive actions. Patients were in panic, were all unhappy, felt an irresistible urge to move, become obsessive and impatient. Usually there is an acute change of mood in the morning and within 2-3 hours suddenly disappeared. Patients with this condition are called a "crisis". Thus patients committed hetero(applied various injuries to closest associates, broke dishes, fist banging on the wall, etc.) and self-injurious behavior (often dissected ulnar vein was applied to the forearm of varying depths and sizes of cuts).

Systematic studies of paroxetine in animals and humans with respect to potential abuse, tolerance and the ability to cause physical dependence have not been conducted. Although clinical studies have not shown the tendency to addiction, these observations were not systematic, and based on such limited results it is impossible to draw conclusions, the extent to which acts on the central nervous system drug abuse cause, will be misused or placed on the market. Therefore, patients must be carefully screened for a history of drug dependence, and its identification should be monitored for these patients, the use of Paxil diversion or abuse (e. g, development of tolerance, increased dosage, drug addiction) [5]. Termination of paroxetine should be done gradually because of the risk of withdrawal symptoms, more frequent than other SSRIs, and is characterized by dizziness, headache, nausea, vomiting, diarrhea, fatigue, unsteady gait, flu-like symptom, anxiety, irritability, insomnia, lethargy, emotional lability, hypomania, tremor, in rare cases, paresthesias and visual impairment [5-8].

However, the data of this work indicate firstly, if not to treat withdrawal symptoms, its duration is longer than three months; 
secondly arising psychopathological disorders are life-threatening.

In the literature known to us, we did not find treatments depending on Paxil and developed a variant based on the existing methods of treatment of psychopathological disorders:

1. Dextrose $5 \%$ ( $5 \%$ solution of glucose for injection) intravenous 1.0 liter + thiamine (vitamin B1) + $250 \mathrm{mg}$ ascorbic acid (vitamin C) + $5.0 \mathrm{~g}$ of furosemide (Lasix ) $2 \mathrm{ml} / 20 \mathrm{mg}+$ diphenhydramine $1 \%-2 \mathrm{ml}$. This mixture was injected intravenously 1 per day (in the afternoon) with a velocity with velocity 60 drops in 1 min for 10 days;

2. Haloperidol $0.5 \%-1.0 \mathrm{ml}+2.5 \%$ pipolfen (prometazine) $-2.0 \mathrm{ml}$ intramuscularly (morning and evening) for 10 days;

3. Chlorpromazine $2.5 \%-2.0$ (or $4.0 \mathrm{ml}$ ) intramuscularly 10 days;

4. Once a week reduced dose of $10 \mathrm{mg}$ paroxetine (Paxil 30-45 days was completely abolished);

5. Carbamazepine 1500-2000 mg per day orally;

6. Lamotrigine (lamictal) $300 \mathrm{mg}$ daily, orally;

7. At night, clonazepam 1-2 mg orally;

8. Amitriptyline $200 \mathrm{mg}$ at day orally.

After 10 days, psychotic (visual and auditory hallucinations) disorders were cropped. However, bouts of dysphoria, dizziness, sensory disturbances, sleep disturbances, anxiety, headache, agitation, nausea, tremor, sweating remained.

Medication therapy, referred to in paragraphs 5-8, held for 45 days. Then, they were administered the following doses: carbamazepine 1200 $\mathrm{mg}$ /day, Lamotrigine $200 \mathrm{mg} /$ day, $1 \mathrm{mg}$ clonazepam overnight per os.

Amitriptyline was continued 3 more months with previous doses. The total duration of treatment was 8.6 months. Thereafter, as the maintenance dose was $100 \mathrm{mg}$ amitriptyline by evening, per os.

\section{Conclusion}

Summing up the results of the study, we can conclude that the main clinical features depending on Paxil are: severe withdrawal symptoms, psychotic phenomena (visual and auditory hallucinations), affective disorders ( depressed mood tinged with gloom, melancholy, gloomy discontent with spiteful irritability); combination of melancholy or sad mood, anxious, irritability, passing in hetero and auto-aggression , suicide attempts. Mood changes usually occur acutely, in the first half of the day and for 2-3 hours suddenly disappeared. Patients called this condition "crisis".

From the data obtained we get inference - Paxil addiction treatment for women should be aimed at eliminating those psychopathological syndromes that are characteristic of this disease. Based on the clinical and psychopathological data patients were assigned to the above therapy.

\section{Funding and other support}

The article carried out by own financial resource.

\section{Author disclosure}

Authors declare that the manuscript is submitted on behalf of all authors. None of the material in this manuscript has been published previously in any form and none of the material is currently under consideration for publication elsewhere other than noted in the cover letter to the editor. Authors declare to have any financial and personal relationship with other people or organizations that could inappropriately influence this work. All authors contributed to and have approved the final manuscript. Conflict of interest: none.

\section{Conflict of interest statement conflict of interest statement}

The authors declare that the research was conducted in the absence of any commercial or financial relationships that could be construed as a potential conflict of interest.

\section{Acknowledgments}

The author would like to thank Dr. Fidan Mamedova for her assistance in improving manuscript editing, as well as the reviewers for their valuable comments and suggestions.

\section{References}

1. Depression Statistics: http://www.lossofsoul.com/DEPRESSION/statistic.htm

2. Bean M (2004) Psychopharmacological and antiepileptic drugs, approved for use in Russia/Ed. S.N. Mosolov 304.

3. WHO. The ICD- 10 Symptom Check list for Mental Disorders World Health Organization in 2004.

4. The ICD-10 (1993) Classification of Mental and Behavioural Disorders. Diagnostic criteria for Research World Health Organization Geneva 262.

5. PAXIL (paroxetine hydrochloride) Tablets and Oral Suspension (2007): PRESCRIBING INFORMATION (PDF). Research Triangle Park, NC: GlaxoSmithKline.

6. Belloeuf L, Le Jeunne C, Hugues FC (2000) Paroxetine withdrawal syndrome. Ann Med Interne (Paris) 151: 399. [Crossref]

7. Black K, Shea C, Dursun S, Kutcher S (2000) Selective serotonin reuptake inhibitor discontinuation syndrome: proposed diagnostic criteria. J. Psychiatry Neurosci 25: 255-261. [Crossref]

8. Rosenbaum JF, Fava ML, Hoog SL, Ascroft RC, Krebs WB (1998) Selective serotonin reuptake inhibitor discontinuation syndrome: a randomized clinical trial. Biol Psychiatry 44: 77-87. [Crossref]

Copyright: (C2016 Aliyev NA. This is an open-access article distributed under the terms of the Creative Commons Attribution License, which permits unrestricted use, distribution, and reproduction in any medium, provided the original author and source are credited. 